\title{
PHYSICO-CHEMICAL DYNAMICS OF NANOPARTICLE FORMATION DURING LASER DECONTAMINATION AND CHARACTERIZATION
}

\section{Research Objectives}

Acceleration of decommission and deactivation (D\&D) activities of US Department of Energy (DOE) requires improved understandings of nanoparticle production and transformation during laser decontamination. Many material researchers have explored particle formation during laser energy-material interaction, but there are little data relevant to D\&D work while laser energy is used to remove materials from contaminated surfaces. Fine particles are harmful to D\&D workers and the environment. The focus of this research is to investigate the effects of the laser parameters and the chemistry of target samples on the dynamics and properties of produced particles. Data will facilitate better design of decontamination and deactivation strategies. There are three goals associated with achieving the required understanding of nanoparticle dynamics.

(1) To develop baseline property data of laser-produced nanoparticles from samples of different bulk chemical composition and surface morphology. The data would include properties of particles such as the size from a few nanometers to a few micrometers, shape, distribution, and chemical composition.

(2) To develop advanced particle instrumentation (for sizing and chemistry measurement) enabling higher resolution and shorter interval measurement, and

(3) To develop computer simulation model to analyze and predict the dynamics of particles produced.

\section{Research Progress and Implications}

This report summarizes work performed for FY2002 (from October 2002 to June 2003). A second harmonic Nd: YAG laser was used as the energy source for material removal. Using the pulsed laser with up to $200 \mathrm{~mJ}$ of energy/pulse, a 3-5 ns pulse width at the wavelength of $532 \mathrm{~nm}$, particles were produced by ablation of the surfaces of target materials, alumina, stainless steel, and concrete. The laser pulses irradiated the surfaces of rotating sample targets placed inside an air leak-tight chamber. A direct assessment of threshold energy for the particle generation by laser ablation has been performed. This is a non-trivial task since it requires the chamber to be particle free prior to an experiment. We had ensured this condition by carefully prepared the sample surfaces the target and the chamber so not a single particle as small as $3 \mathrm{~nm}$ could be detected by our sensitive instrument before we conducted an experiment. Using the instrument, an ultrafine condensation particle counter, the generated particle concentrations were examined with respect to laser energy. Three target samples made of concrete, stainless steel (316), and alumina were used, respectively. Laser shots having a certain energy value were counted. Sets of correlations between the number of shots and the generated particle concentrations were examined. A correlation for alumina target is shown below. For alumina target, the threshold laser fluence is $1.7 \mathrm{~mJ} / \mathrm{cm}^{2}$, while those for stainless steel and concrete are 3.0 and $12.4 \mathrm{~mJ} / \mathrm{cm}^{2}$, respectively. The diameter of the laser beam was $6 \mathrm{~mm}$, and the threshold laser energy for each pulse were $0.487,0.86$, and $3.5 \mathrm{~mJ}$ for alumina, stainless steel, and concrete, respectively.

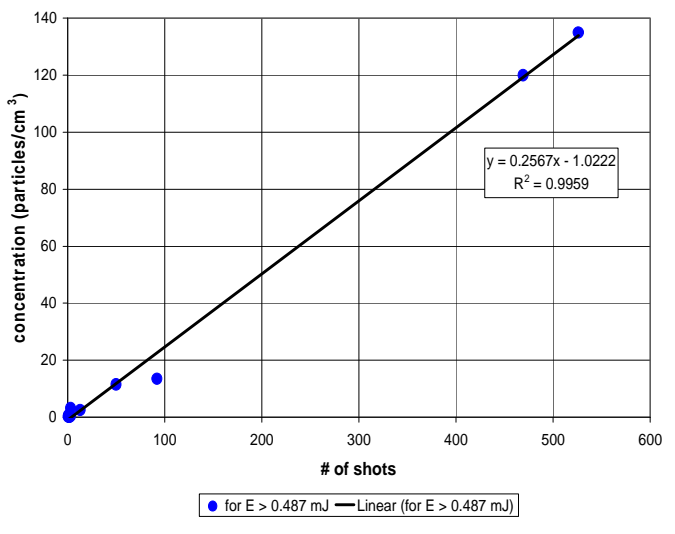

We found that, in general, the higher total laser energy (i.e., in a single shot or totaled in a number of weaker shots in a given time) used, the greater the total number concentrations of particles yielded. The peak size (i.e., diameter) of the generated particles generally remained in between 100 and $200 \mathrm{~nm}$ with smallest diameter we measured around $2 \mathrm{~nm}$ and largest in micrometer size range depending upon experimental conditions. The variation of the peak size for a given energy level was larger at larger laser energy. Two sources of particle formation were 


\section{PHYSICO-CHEMICAL DYNAMICS OF NANOPARTICLE FORMATION DURING LASER DECONTAMINATION AND CHARACTERIZATION}

identified that are (1) direct formation by the laser (i.e., the primary particles) and (2) formation through coagulation/ coalescence from the seedling nuclei during the first few seconds or faster (i.e., the secondary particles). The dispersion of the size distribution was relatively constant at a value of approximately three. Higher laser energy provided much more nanoparticles, those smaller than $50 \mathrm{~nm}$ in diameter, than that produced by lower energy. The number concentrations produced under various laser energy conditions were found to be generally non-linear. The relationship between the number of particles produced and energy deposition frequency varying from 1 shot in every 150 seconds to $10 \mathrm{~Hz}$ (1500 shots in 150 seconds) were also found to be non-linear and have a threshold at the lower end using the fundamental, the second harmonic, and the $4^{\text {th }}$ harmonic wavelengths of the laser. Co-PI, Dr. Da-Ren Chen of Washington University (WashU) in St. Louis, has completed the design and sent the drawing to a machine shop for fabrication in May 2002. This novel stack differential mobility array design is brand new and not available on the commercial market. The lead PI and Dr. Chen are currently filing a joint Intellectual Property disclosure through the Technology Transfer Office at ORNL. The design enables a great flexibility in configuration, operation, and rapid measurement for a wide range of nanoparticle sizes. The fabrication was completed in December 2002. Laboratory tests are ongoing to obtain the instrument transfer function and measurement efficiency. Also investigated are the instrument reliability and duty cycle.

\section{Planned Activities}

For this fiscal year, we will finish the experiments using a second harmonic (i.e., $532 \mathrm{~nm}$ ) and part of the work by other wavelengths (i.e., 1064 and $266 \mathrm{~nm}$ ). Results of the first year have been presented at the annual meeting of the American Association for Aerosol Research (AAAR) in October 2002. The preliminary results of this year will be compared with that of last year and presented in the upcoming AAAR meeting in October 2003 and in an invited American Chemical Society meeting in September 2003. For the rest of the fiscal year (FY2003), an electrostatic precipitator built in 2002 will be employed to collect the nanoparticles for SEM/TEM analysis for morphology analysis. Time-resolved laser-induced breakdown spectroscopy will be used to analyze, on-line, the chemical composition of particles generated. Combining the particle size information, we would be able to produce the size distribution of various metals embedded in the particles. Chromium-doped target samples will also be used in 2003. The new DMA being developed at WashU will be employed to probe the particle dynamics on a shorter time scale (sub milliseconds). This will require reconfiguration of a window on the current aerosol chamber to house an equilibrium conduit for hosting the new DMA. Control electronics and software for running the DMA will also be developed and tested. Individual modules of the simulation model will be developed and/or collected from various sources and debugged. Initial runs will be made and the results will be compared to the data generated in 2002 and 2003. During the final fiscal year (FY2004), similar experiments to those conducted in FY2002 and 2003 will be performed using radionuclide-doped target samples. After all the computer program modules for simulating particle dynamics are completed, the overall program will be evaluated. Prognosis using the simulation model will be undertaken. A particle control strategies and engineering design will be developed.

\section{Information Access}

Yining Lin, a postdoctoral working with the PI and the PI has submitted a paper to be presented in the Annual Meeting of the American Association for Aerosol Research in October. A novel aerosol spectrometer developed by the PI in an earlier project has been awarded a US patent \# 6,359,687 in April 2002. This technology will be used in the second year to measure the composition of particles generated. Dr. Doh-Won Lee, a new postdoctoral, started work with the PI at ORNL in November 2002. IP disclosure is currently (in June 2003) being filed through ORNL to DOE Head Quarter. 